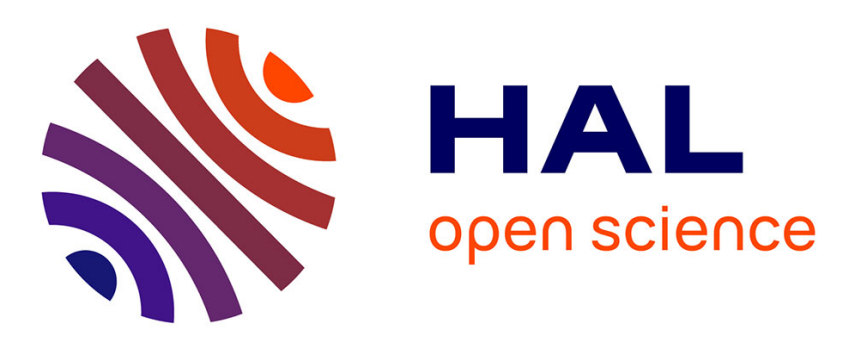

\title{
Un autre regard sur l'archaïsme dans les sépultures privées de l'Égypte pharaonique
}

\author{
Héloïse Smets
}

\section{To cite this version:}

Héloïse Smets. Un autre regard sur l'archaïsme dans les sépultures privées de l'Égypte pharaonique. Archimède: archéologie et histoire ancienne, 2021, Archimède. Archéologie et histoire ancienne, 8 , pp.117-127. 10.47245/archimede.0008.ds2.03 . halshs-03279823

\section{HAL Id: halshs-03279823 \\ https://shs.hal.science/halshs-03279823}

Submitted on 6 Jul 2021

HAL is a multi-disciplinary open access archive for the deposit and dissemination of scientific research documents, whether they are published or not. The documents may come from teaching and research institutions in France or abroad, or from public or private research centers.
L'archive ouverte pluridisciplinaire HAL, est destinée au dépôt et à la diffusion de documents scientifiques de niveau recherche, publiés ou non, émanant des établissements d'enseignement et de recherche français ou étrangers, des laboratoires publics ou privés. 
1 DOSSIER THÉMATIQUE 1

SIUE DEUS SIUE DEA. DÉNOMINATIONS DIVINES DANS LES MONDES GREC ET SÉMITIQUE : UNE APPROCHE PAR LE GENRE

DOSSIER THÉMATIQUE 2

TRADITION ET TRANSMISSION DANS L'ANTIQUITÉ : RÉFLEXIONS INTERDISCIPLINAIRES

98 Claire CAMBerlein, Efstathia Dionysopoulou \& Thibault Foulon

Introduction. La tradition et sa transmission : positionnements théoriques

105 Mélissa Leuzy

Consigner ou fabriquer la légende d'Alexandre le Grand ? Arrien face à la tradition dans l'Anabase

\section{Héloïse SMETS}

Un autre regard sur l'archaïsme dans les sépultures privées de l'Égypte pharaonique

128 Laura WALDVOGEL

Nouveaux indices de variabilité au sein des traditions funéraires de l'ouest du Rubané occidental

141 Grégoire BLANC

Praxis citationnelle et co-construction du discours scientifique dans les Naturales Quaestiones de Sénèque (livres II \& III)

150 Anthony GLAISE

Polémiques, traditions et identités : réflexions autour des Discours contre les juifs et les judaïsants de Jean Chrysostome

159 ACTUALITÉ DE LA RECHERCHE

QUOI DE NEUF À L'OUEST DE STRASBOURG ? KOENIGSHOFFEN : ÉTAT DES LIEUX ET DÉCOUVERTES RÉCENTES

195 VARIA 


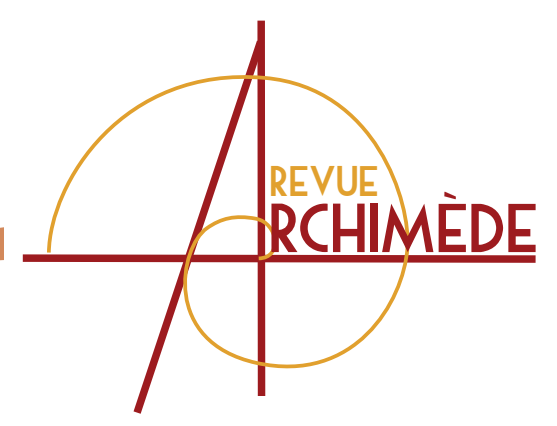

ARCHÉOLOGIE ET HISTOIRE ANCIENNE

\title{
UN AUTRE REGARD SUR L'ARCHAIISME DANS LES SÉPULTURES PRIVÉES DE L'ÉGYPTE PHARAONIQUE*
}

\author{
Héloïse SMETS \\ Doctorante en égyptologie \\ Université de Strasbourg \\ UMR 7044 Archimède \\ h.smets@unistra.fr
}

\begin{abstract}
* Cet article est réalisé dans le cadre de ma thèse « Archaïsme et citations artistiques dans les sépulture privées de la nécropole thébaine du Nouvel Empire à la Basse Époque (1550 - 332 av. J.-C.) » menée à I'Université de Strasbourg sous la direction du professeur Frédéric Colin et initialement consacrée à l'étude de la TT 388 grâce à la généreuse proposition du professeur Claude Traunecker.
\end{abstract}

\section{RÉSUMÉ}

L'archaïsme est aujourd'hui conçu en égyptologie comme un retour volontaire au contenu culturel du passé après une rupture de la chaîne de transmission continue de celui-ci. Toutefois, le phénomène décrit par cette définition est plus complexe et la terminologie actuelle de I'archaïsme demeure insuffisante. Afin de mieux comprendre et caractériser

\section{MotS-CLÉs}

Art égyptien,

archaïsme,

tradition,

innovation,

citation artistique,

scènes de boucherie,

procession d'offrandes. cette forme de réinterprétation de la tradition artistique, nous proposons ici de considérer l'archaïsme des tombes privées comme l'une des formes que prend la citation artistique en Égypte pharaonique.
Archaism is currently understood in Egyptology as a voluntary return to the past's cultural content after a break in the chain of its continuous transmission. However, the phenomenon described by this definition is more complex and the current terminology of archaism remains insufficient. To better understand and characterize this form of reinterpretation of the artistic tradition, we propose here to consider the archaism of private tombs as one of the possible forms of artistic quotation in Pharaonic Egypt.

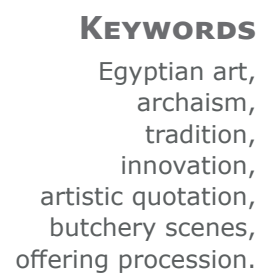




\section{TRADITION ET ARCHAÏSME}

La tradition est très présente dans l'art égyptien pharaonique, car les représentations suivent le canon formel de l'image du roi et des dieux fixé dès la fin de l'époque prédynastique. Un répertoire commun général et les formes qui y sont associées sont ainsi transmis à travers les millénaires. Lorsqu'un artiste égyptien réalise un décor, il puise dans ce répertoire et en respecte les conventions. De cette forte fidélité à la tradition résulte un aspect immuable de l'art égyptien, en dépit des évolutions stylistiques et des modifications de la grille de proportions [1]. L'art pharaonique a dès lors souvent été considéré comme dénué de créativité et qualifié de reproductif, en opposition à I'art productif [2].

Au sein de cette longue transmission continue, des hiatus peuvent néanmoins survenir. Dans le cadre de troubles politiques et de périodes économiquement difficiles par exemple, une partie du contenu culturel peut disparaître et être ensuite redécouvert et réutilisé. On parle alors généralement d'archaïsme et d'art archaïsant.

Attesté depuis Denys d'Halicarnasse (I ${ }^{\text {er }}$ siècle

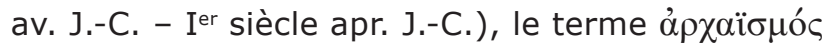
décrivait à l'origine le charme du style ancien et, par extension, I'utilisation en littérature d'une expression obsolète [3]. Aujourd'hui, dans l'usage courant, l'archaïsme définit le caractère d'ancienneté que peut posséder un objet, un site, une langue ou encore un art. Dans le domaine artistique en particulier, il s'agit de «l'imitation de la manière (...) et des procédés des œuvres anciennes » [4].

[1] LABOURY 2017, p. 230.

[2] Sur la nuance entre productif et reproductif, voir WinAND 2017, p. 21-40.

[3] Denys d'Halicarnasse, Traité de l'arrangement des mots, 22.

[4] Centre National de Ressources Textuelles et Lexicales (CNRTL), https://www.cnrtl.fr/definition/archaïsme, lien consulté le 14/05/2020.

[5] Ibid.

[6] Voir, par exemple, Les peuples à civilisation archaïque face au monde moderne, de François Honti dans le Monde diplomatique, https://www.monde-diplomatique. fr/1966/01/HONTI/27052, lien consulté le 14/05/2020.
En rhétorique, il désigne l'utilisation « de mots ou de tournures tombés hors de l'usage général » et acquiert ainsi souvent une connotation négative [5]. On a par ailleurs longtemps opposé en sociologie les sociétés modernes aux sociétés ou peuples archaïques, ces derniers étant considérés comme arriérés parce qu'ils n'ont pas connu le même développement que les sociétés occidentales et non isolées [6]. De nos jours, on préfère parler de peuples isolés indigènes ou non contactés [7], mais il faut noter que l'archaïsme, et en particulier ses adjectifs, ont encore souvent une signification péjorative, même en dehors des domaines mentionnés ici [8].

En histoire de l'art et en archéologie grecque, on différencie généralement l'archaïsme de l'art archaïsant. Le premier est d'ordre strictement chronologique, car il définit la période grecque archaïque qui s'étend de 650 à 480 av. J.-C. Quant au second, qui nous intéresse ici, c'est l'art « qui, en Grèce et dans le monde romain, se réfère volontairement et consciemment à l'art archaïque grec et en reproduit les principales caractéristiques »[9], notamment pour affirmer une certaine identité ou donner un argument d'autorité dans le cadre d'un certain conservatisme [10].

Selon Valérie Huet et François Lissarrague, est donc « archaïque » ce qui appartient à une période bien précise de la chronologie relative grecque, tandis qu'est « archaïsante » ou « pseudo-archaïque » toute œuvre qui reprend volontairement des éléments du passé, spécifiquement de la période archaïque [11], pour obtenir un effet particulier [12]. Et c'est la notion de volonté qui distingue pour eux l'art archaïsant de la survivance [13]. Nous verrons qu'il en est autrement en égyptologie.

Sur les termes « archaïque » et « primitif » en ethnologie, voir LÉVI-STRAuss 1952, p. 3 et p. 18-20.

[7] Voir, par exemple, https://survivalinternational.fr/ peuplesnoncontactes, lien consulté le 14/05/2020.

[8] BRuit Zaidman \& GHeRchanoc 2006, p. 5.

[9] ZaGDOUN 1989, p. 11.

[10] BRUit Zaidman \& GHERCHANOc 2006, p. 8-9.

[11] Lorsque l'art se réfère à l'époque classique, il sera dit « classicisant ». Huet \& Lissarrague 2006, p. 182. Ces deux styles constituent des déclinaisons de l'art « néo-attique ». Voir DARDENAY 2013, p. 123.

[12] HUET \& LisSARRAGUe 2006 , p. 179-182.

[13] Ibid., p 180. 
Alexandre Grandazzi, spécialiste de l'archéologie romaine, rappelle que I'archaïsme renvoie également à la périodisation dans sa discipline. En effet, pendant longtemps, l'adjectif « archaïque » se référait uniquement à la toute fin de la Royauté et au début de la République, c'est-à-dire le dernier tiers du VIe siècle et le $V^{e}$ siècle av. J.-C. à Rome. Ensuite, la fourchette chronologique a intégré le début de la Royauté [14].

Contrairement à V. Huet et F. Lissarrague, A. Grandazzi ne fait pas de distinction entre « archaïque » et « archaïsant » et affirme que sont archaïques à la fois ce qui est ancien et ce qui se fait passer pour ancien [15]. En revanche, il propose deux dénominations : I'archaïsme de conservation et l'archaïsme de création. Le premier signifie qu'on perpétue l'ancien, comme une survivance, tandis que le second consiste à produire du neuf en lui donnant l'apparence de l'ancien, ce qu'on peut également appeler une fabrication du passé [16].

\section{L'ARCHAÏSME EN ÉGYPTOLOGIE}

Le terme « archaïsme » a été transposé de l'archéologie classique à l'égyptologie dès le début du XX siècle. Henri Gauthier l'adopte par exemple dans le Précis de l'histoire d'Égypte par divers historiens et archéologues en 1932 [17]. L'archaïsme et ses termes dérivés vont ainsi cohabiter, puis prendre le pas sur les dénominations revival, restoration ou « renaissance». Celles-ci étaient en usage depuis I'ouvrage d'Heinrisch Brugsch de 1877 [18] pour désigner le phénomène de l'imitation du passé constaté dans l'art, l'architecture, les textes et la religion de la $26^{\mathrm{e}}$ dynastie saïte.

Dans ses premières utilisations, l'archaïsme en égyptologie était considéré comme l'apanage de cette dynastie et était la plupart du temps le reflet d'une vision péjorative de celle-ci. Ainsi, on décrivait l'art saïte comme archaïsant et « amolli » [19], qui manquait de conviction intérieure et se caractérisait par une importance du style au détriment du contenu [20].
Comme l'exprimait Walther Wolf, « Die Rückbesinnung auf eine klassische Epoche zeitigt zwar den Willen, alte Formen neu zu beleben, vermag sie aber nicht mit dem Sinngehalt zu erfüllen, der sie einst geschaffen hatte. So bleiben sie leere, tote Gehäuse » [21].

Progressivement, les limites chronologiques du phénomène sont repoussées. Les égyptologues considèrent alors que les dirigeants kouchites de la $25^{e}$ dynastie (environ 760 - 653 av. J.-C.), d'origine étrangère, en sont les précurseurs dans le cadre d'une politique de légitimation [22]. Ensuite, dès le début des années 1960, Cyril Aldred propose de faire remonter l'archaïsme à la Troisième Période Intermédiaire (environ 1069 - 712 av. J.-C.) en se fondant sur l'étude de plaques en faïence dont le style et les proportions reproduisent ceux de I'Ancien Empire (environ 2700 - 2181 av. J.-C.) [23].

En réalité, l'intérêt pour le passé et sa réutilisation sont omniprésents dans la tradition égyptienne, et l'archaïsme peut apparaître à toutes les périodes. Ce constat est fait en 1972 par Richard Fazzini, qui précise que le phénomène est exacerbé à la Basse Époque, mais pas exclusif à celle-ci [24], et que parmi les raisons d'imiter un monument, il ne faut pas négliger sa proximité géographique et l'admiration pour son esthétique [25].

Aujourd'hui, la définition de l'archaïsme en égyptologie a donc une emprise chronologique plus vaste et elle est également plus neutre [26] : c'est un « conscious return [27] to the cultural modes of the past that are not linked to the time of reappropriation through a traditional transmission » [28]. Les deux éléments clés de cette définition sont donc l'aspect volontaire et l'impératif d'un hiatus dans la chaîne de transmission. L'archaïsme est ainsi mis en parallèle avec le renouveau (revival) et considéré comme l'antonyme de la tradition continue, du conservatisme ou d'une survivance (survival) [29]. Selon cette définition, il est dès lors nécessaire d'être en mesure de prouver que la transmission de la tradition en question a été interrompue
[14] Grandazzi 2006, p. 90.

[15] Ibid., p. 91.

[16] Ibid., p. 89.

[17] GAUTHIER 1932, p. 232.

[18] BRUGSCH 1877, p. 739.

[19] MASPERO 1887, p. 178.

[20] ALDRED 1961, p. 156.

[21] WoLF 1930, p. 269.

[22] Kees 1953, p. 198 ; Bоthmer \& Riefstahl 1960, p. Xxxvii.

[23] AldRED 1961, p. 155.

[24] FAZZINI 1972, p. 67-69.

[25] Ibid., p. 68.

[26] Par exemple : Valbelle 2005, p. 184.
[27] Malgré ce qu'en dit Hellmut Brunner dans le Lexikon der Ägyptologie. Bien qu'il ait été rédigé dans les années 70 , la vision qui y transparaît est assez ancienne. Voir BRUNNER 1975.

[28] JURMAN 2010, p. 76.

[29] LABOURY 2013, p. 12, n. 5. Par exemple, Morkot 2003, p. 79-81; Der Manuelian 1998, p. 231 ; Davis 2003, p. 31 ; KAHL 2010, p. 1. La survivance (survival) est le « fait qu'une institution, qu'une pratique sociale ou culturelle se maintienne après la disparition des circonstances qui I'ont produite, sous une forme affaiblie, marginale ou stéréotypée ». Centre National de Ressources Textuelles et Lexicales, https://www.cnrtl.fr/definition/archaïsme, lien consulté le 14/05/2020. 
avant de réapparaître si I'on veut identifier un cas d'archaïsme [30]. Cela peut s'avérer ardu dans la mesure où les vestiges du passé ne nous sont bien entendu pas tous parvenus, et que leur absence ne signifie pas d'emblée que la tradition a cessé d'exister [31].

On remarquera ici que, bien que le terme « archaïsme » ait été emprunté à l'archéologie classique, il n'en a pas conservé exactement le même sens. En effet, la distinction entre archaïsme et survivance a été établie à partir d'un critère différent : la présence ou l'absence de conscience du phénomène en archéologie classique et la présence ou l'absence d'un hiatus dans la tradition en égyptologie [32]. Par ailleurs, I'archaïsme a une extension chronologique beaucoup plus vaste en égyptologie qu'en archéologie classique. Il est important de garder ces différences à l'esprit pour éviter les mécompréhensions dans le cadre de dialogues entre disciplines.

\section{UN CONCEPT QUI RESTE PROBLÉMATIQUE}

Malgré la présence du concept en égyptologie depuis plusieurs siècles, l'élargissement de son extension chronologique et l'objectivation de sa définition, I'archaïsme n'a pas été réellement théorisé, à l'exception des pistes proposées par Claus Jurman [33]. Le phénomène doit encore être exploré et la terminologie pose problème à plusieurs égards.

Le premier problème consiste en un manque d'homogénéité de la terminologie dans la bibliographie liée à ce concept, en particulier lors du passage d'une langue à une autre [34]. En anglais par exemple, on rencontre trois adjectifs : archaic, archaizing et archaistic. Archaic fait au départ référence aux premières dynasties pharaoniques, mais il a parfois été utilisé à tort dans le sens d'archaizing, I'adjectif approprié du terme archaism.

Bernard von Bothmer, spécialiste de la sculpture de la Basse Époque, distinguait le terme archaizing, qu'il réservait à la 26e dynastie, de celui d'archaistic qu'il utilisait pour décrire l'art de la $30^{e}$ dynastie qui s'inspirait de la $26^{e}$. Il regroupait par ailleurs ces deux termes

[30] Comme l'explicite notamment PischIkova 2014, p. 73. [31] JURMAN 2010, p. 80.

[32] À l'exception de LABOURY 2013, p. 23, qui distingue l'archaïsme de la survivance par la conscientisation du phénomène, comme en archéologie classique.

[33] JURMAN 2010.

[34] Le même constat a été fait par JuRMAn 2010, p. 75. Ce problème lié à la langue est aussi présent en archéologie classique, comme l'ont fait remarquer HUET \& LISSARRAGUE 2006 , p. 80.

[35] BOthMER \& Riefstahl 1960, p. xxxvii. dans la catégorie des classicismes [35]. Il employait également le terme neo-archaism à propos de l'art de la 30 dynastie [36]. Cette différenciation terminologique entre ces deux dynasties n'a pas lieu d'être à partir du moment où l'on reconnaît que l'archaïsme peut se manifester à toutes les époques de I'histoire pharaonique. C'est probablement la raison pour laquelle beaucoup d'égyptologues ont ensuite utilisé les termes archaizing et archaistic sans distinction.

Les publications en langue anglaise emploient par ailleurs la dénomination revival pour désigner l'archaïsme, en opposition à survival qui correspond à la tradition continue [37]. Néanmoins, dans l'Oxford Encyclopedia of Ancient Egypt, Jack Josephson ne procède pas à une telle distinction. Pour lui, ces deux termes sont des synonymes et l'archaïsme constitue « the survival or presence of something from the past that was not necessarily preconceived $\gg[38]$. Il y englobe donc tous les éléments du passé qui sont encore présents, sans différencier ni les cas conscients de ceux qui ne le seraient pas [39] ni la tradition continue de celle qui est interrompue avant d'être reprise. Cette définition trop large ne permet pas une compréhension fine des phénomènes d'utilisation du passé dont fait partie l'archaïsme.

L'allemand est plus cohérent en utilisant le substantif Archaismus, I'adjectif archaistisch et le participe présent adjectivé archaisierend. Certains chercheurs allemands ont toutefois proposé des termes de substitution à Archaismus sur lesquels nous reviendrons plus tard. En français, I'appellation « renaissance » (saïte, kouchite ou éthiopienne selon les auteurs) a eu un grand succès et a parfois été transposée en anglais [40]. Elle a été beaucoup moins utilisée en allemand [41] et on ne la rencontre presque plus aujourd'hui dans les publications consacrées à l'archaïsme à la suite d'une forte critique [42].

Au départ, cette critique procédait d'une vision péjorative de la Basse Époque. Par exemple, Adolf Erman affirmait que cela ne relevait pas d'une renaissance, mais seulement du mouvement malsain d'une époque qui tente de revenir à une éducation supérieure passée [43]. Les chercheurs étaient alors fortement

[36] BOTHMER 1985, p. 101.

[37] Par exemple, KaHL 2010, p. 1 ; DAVIS 2003, p. 31.

[38] JOSEPHSON 2001, p. 109.

[39] Ce constat a été fait par JURMAn 2010, p. 75.

[40] Par exemple, TIRADRITTI 2008. À ce sujet, voir YoyotTE 1987 , p. 75.

[41] NeUReiter 1994, p. 221.

[42] Elle est néanmoins encore très présente dans le titre de I'exposition Egyptian Renaissance: Archaism and the Sense of History in ancient Egypt. Voir TIRADRITTI 2008. [43] Wolf 1930, p. 268-269 ; ERMAN 1934, p. 321. 
influencés par leur temps, en particulier par le nationalisme des $\mathrm{XIXe}$ et $\mathrm{XX} \mathrm{X}^{\mathrm{e}}$ siècles, ce qui les a conduits à déprécier tout ce qui ne semblait pas correspondre à l'idée d'une grande nation [44]. Si le terme « renaissance » a été rejeté dans la deuxième moitié du $X X^{e}$ siècle, c'était d'abord pour éviter un anachronisme avec la Renaissance du Quattrocento et la Renaissance française [45], et ensuite parce qu'il ne rend pas compte des caractéristiques spécifiques du phénomène en question, comme la réutilisation simultanée de modèles de plusieurs époques dans certains cas [46].

La question de l'hétérogénéité de la terminologie s'avère importante, non seulement car elle peut porter à confusion pour toute personne s'intéressant au sujet ou communiquant sur celui-ci, mais aussi parce que, comme l'a remarqué Claus Jurman, la définition des termes utilisés a une répercussion sur les résultats directs de la recherche [47].

\section{L'APPORT DES SCÈNES DE BOUCHERIE ET DE PROCESSIONS D'OFFRANDES}

Le deuxième problème de la définition actuelle de I'archaïsme est qu'elle présente une lacune que I'on peut notamment constater par l'étude des scènes de boucherie [48] et de processions d'offrandes au défunt dans les tombes thébaines de la Basse Époque, comme nous allons le voir.

En effet, des éléments du décor de la plupart des grandes tombes de l'Assasif, telles $T 27$ (Chéchonq), TT 34 (Montouemhat), Tा 36 (Ibi), TT 37 (Haroua), TT 279 (Pabasa), TT 312 (Nespakachouty), TT 389 (Basa) et également de la TT 388 (Anonyme), s'inspirent entre autres du décor de la chapelle d'Hatchepsout située dans son temple de Deir el-Bahari [49]. Il s'agit d'une petite pièce consacrée au culte de la reine et située sur la terrasse supérieure du temple. Les scènes de boucherie s'étendent sur la paroi est, en miroir de part et d'autre de l'entrée. La scène de la découpe de la patte avant du bovin sacrifié y est démultipliée sur trois registres. Les

[44] BRUNNER 1970, p. 156-157.

[45] SPALINGER 1978, p. 12.

[46] Eigner 1984, p. 17-18.

[47] JURMAN 2010, p. 74-75.

[48] Sur les scènes de boucherie, voir EgGEBRECHT 1973.

[49] Le premier à avoir identifié ce lien est ERMAN 1914.

[50] Voir NAVILLE 1901, pl. CVII-CXIII.

[51] Der Manuelian 1994, p. 33-35 et p. 44, fig. 11

[52] Pischikova 1998, p. 67.

[53] Son plan et quelques brèves descriptions ont été publiés dans PM I, p. 439 et FAKHRY 1947, p. 34. Claude

Traunecker est le premier à approfondir la description et le sujet dans TRAUNECKER 2014, p. 223. processions de porteurs se déroulent quant à elles en parallèle sur les parois nord et sud qui se terminent par la représentation de la reine et la liste des offrandes [50]. Dans la TT 34 de Montouemhat, la composition des trois registres superposés de boucherie a été reprise mais redécoupée de sorte qu'ils soient disposés en un seul registre bas [51]. Dans la TT 312 du vizir Nespakachouty, creusée à même la falaise rocheuse près du temple d'Hatchepsout, ce sont à la fois les scènes de boucherie et de procession d'offrandes de la chapelle qui ont servi d'inspiration [52]. Il en va de même pour la tombe thébaine 388, non étudiée à ce jour [53], dont la seule pièce décorée comporte trois registres superposés de scènes de boucherie sur la paroi est et des processions de porteurs sur les parois nord et sud. De nombreux détails, tels que la démultiplication de la découpe de la patte avant, le contenu des offrandes, ou encore la forme des cornes des animaux, permettent d'identifier les liens entre ces représentations et celles de la chapelle d'Hatchepsout [54], sans que I'on puisse dire qu'elles sont totalement identiques [55].

Le concept actuel d'archaïsme ne s'avère donc pas suffisant pour qualifier ces représentations. Ce type de scène est bel et bien absent des sépultures depuis le Nouvel Empire jusqu'à la Basse Époque et le monument qui sert ici de modèle est certes plus ancien. Toutefois, force est de constater que ce dernier est également géographiquement proche des sépultures qu'il inspire, à quelques centaines de mètres seulement. Il est par ailleurs très présent dans le culte local et encore régulièrement fréquenté au moment de la conception des tombes concernées [56]. En outre, les décors de ces sépultures contemporaines sont également inspirés les uns des autres dans de nombreux cas [57]. Par conséquent, bien que le rapport au passé soit fondamental dans ces décors, il ne faut négliger ni l'importance que peut avoir la proximité géographique dans de nombreux cas [58] ni leur ancrage dans le présent. Or, ces deux facteurs, en particulier le second, ne sont pas pris en compte lorsqu'on utilise le concept d'archaïsme pour caractériser ces représentations.

[54] Voir notamment pour la TT 312 : PISCHIKovA 1998, p. 70-73.

[55] Ces observations à propos de la TT 388 ont été rendues possible par le professeur C. Traunecker qui m'a aimablement donné accès à son dossier la concernant. Je I'en remercie tout particulièrement.

[56] En témoigne le fait que ces sépultures possèdent des couloirs d'accès qui démarrent à l'emplacement de l'allée processionnelle du temple.

[57] Par exemple, la TT 34 a notamment inspiré la TT 312, voir infra.

[58] Comme le remarquait déjà FAzZini 1972, p. 98. Voir également Perdu 2018, p. 217. 


\section{ARCHAÏSME ET INNOVATION}

Par ailleurs, le terme d'archaïsme tel qu'il est conceptualisé actuellement implique d'une part un modèle ancien et d'autre part sa copie, qu'elle soit considérée comme directe ou indirecte. Cela procède d'une vision foncièrement linéaire qui n'admet pas la présence de créativité dans ce processus.

Dans I'usage courant, I'appellation « copie » signifie en effet la réalisation d'une reproduction fidèle en tous points au modèle. Or, il n'existe pas deux copies exactes dans l'art égyptien. Chaque nouvelle représentation, y compris dans les scènes « archaïsantes », implique l'introduction de nouveaux éléments ou de modifications qui en font une innovation [59]. Même si ces modifications peuvent sembler mineures, le déplacement d'une scène au sein d'un nouvel espace et son adaptation à celui-ci signifient déjà que son sens peut en être modifié [60]. En effet, selon sa position dans la tombe ou son étendue, l'image ne sera pas perçue de la même manière par son récepteur et sa fonction peut changer.

Dès lors, il faut éviter les termes « copie » et « itération » [61], et au lieu d'opposer la tradition et I'innovation, il nous faut aborder l'art égyptien en considérant que la tradition constitue une base à l'innovation [62]. De même, cela atteste qu'il ne s'agit pas d'une tradition strictement reproductive, mais productive, dans le sens d'une tradition soumise «à une adaptation déterminée par les exigences de la réalité contemporaine $\gg[63]$.

De la même manière, plutôt que de se contenter de copier la tradition dans une démarche statique, l'archaïsme en art égyptien est un processus dynamique [64] qui crée un nouveau produit culturel en réactualisant, réinterprétant et adaptant à un nouveau contexte le contenu culturel du passé.

En effet, aucun des exemples susmentionnés n'est identique à un autre et chacun résulte de différents choix, que ce soit celui des éléments sélectionnés ou celui de la disposition de ces derniers. István Nagy

[59] LABOURY 2017, p. 232-238.

[60] FORERO-MENDOZA 2004, p. 21-27

[61] Il s'agit d'un terme utilisé par ENGERT 2015 tout au long de sa publication. Elle y adjoint la notion de déviation ou d'écart pour pouvoir rendre compte des différences qui existent entre les itérations et leur modèle. ENGERT 2015 , p. 373.

[62] LABOURY 2013, p. 24-25.

[63] JURMAN 2010, p. 78.

[64] Voir DEN DONCKER 2017, p. 335

[65] NAGY 1973, p. 63.

[66] AldRED 1980, p. 222.

[67] NaVRATILOVA 2017, p. 555. avait entamé cette réflexion en 1973 en affirmant que les cas d'archaïsme ne sont parfois pas des copies serviles [65] et Cyril Aldred écrivait quelques années plus tard qu'il s'agissait de réinterprétations [66].

Toutefois, cela ne transparaît pas dans la terminologie actuelle. Les égyptologues ont conscience que le terme pose problème sur ce point et utilisent par exemple I'expression inventive ou innovative archaism [67]. De même, la locution archaism and innovation que l'on retrouve souvent dans les titres des publications [68] souligne la présence de l'innovation aux côtés de l'archaïsme. Cependant, l'innovation devrait en réalité être incluse à la notion d'archaïsme elle-même, et ces tournures montrent bien qu'il y a là un manque. Dans la plupart des cas, le terme « archaïsme » se trouve entre guillemets [69], sans doute pour se distancier par rapport à la connotation péjorative que peut encore avoir cette dénomination, mais cela ne paraît pas suffisant [70].

Certains ont utilisé des termes différents pour parler de l'archaïsme à la Basse Époque, tels que Sabine Neureiter et Jan Assmann, qui emploient respectivement Revitalisierung et Repristination [71]. Ces deux termes sont intéressants, notamment car ils tiennent compte de l'aspect conscient et volontaire du processus et ne portent pas de connotation négative. Mais ils correspondent tous deux à l'idée du retour d'une tradition ancienne, sans considérer l'innovation à partir de cette tradition. Il en va de même pour le terme Rückgriff utilisé par Alessandro Roccati et Michaela Engert [72].

\section{UNE NOUVELLE APPROCHE DE L'ARCHAÏSME COMME FORME DE CITATION ARTISTIQUE}

Plutôt que de chercher un terme de remplacement à l'archaïsme, nous tenterons ici d'aller au-delà en proposant de l'intégrer au concept de citation artistique, ce qui permet d'être plus proche de la réalité de ce phénomène quand il concerne les décors des tombes privées.

[68] Par exemple, Silverman 2009 ; MoRKot 2007 ; TIRADRITTI 2013 ; PISCHIKova 2014, p. 73. Cela n'est pas sans rappeler la dénomination « archaïsme de création » d'Alexandre Grandazzi. Voir supra.

[69] Par exemple, JURMAn 2009, p. 129 ; WiLson 2010, p. 241 et p. 253. On peut constater la même réserve à propos du terme copy chez Der MANUELIAN 1994, p. 51 et de l'expression pharaonic renaissance chez TIRADRITTI 2013, p. 18.

[70] Un constat émis également par LABOURY 2013, p. 11. [71] AsSMANN 1992, p. 181 et p. 195.

[72] Roccati 1995 et ENGert 2015. Pour une vision synthétique sur I'historiographie de I'archaïsme en égyptologie, voir StAMMERS 2009, p. 83-88 et JURMAN 2010. 
La citation artistique, au contraire de l'acception usuelle du terme « citation », n'équivaut pas à une stricte répétition. Il s'agit de s'inspirer ou de réutiliser une image existante, mais en la réactualisant par sa transposition dans un autre espace et en la réinvestissant d'un message nouveau [73]. Citer artistiquement implique donc de la créativité. Ce concept théorisé dans I'histoire de l'art contemporain s'applique très bien à l'archaïsme dans les tombes privées égyptiennes.

À titre d'exemple, le célèbre décor de la tombe du vizir Rekhmirê (TT 100) est une citation artistique du décor de la sépulture de son oncle et prédécesseur Ouseramon (TT 131). L'ensemble de ce programme décoratif a été repris, mais chaque scène a été déplacée à un autre endroit et enrichie de nombreux détails nouveaux qui en font une œuvre unique. Il s'agit donc bien ici d'une citation qui concerne deux monuments très proches géographiquement et dont les défunts sont liés professionnellement et familialement [74]. Mais il ne s'agit pas d'archaïsme, puisqu'elles sont contemporaines. On trouve également des cas de citations entre tombes contemporaines à la Basse Époque, par exemple avec la scène de porteurs d'offrandes des parois est et ouest de la tombe de Nespakachouty (TT 312) qui cite celle de la sépulture de Montouemhat (TT 34) en transformant certains porteurs en porteuses [75].

Ainsi, il serait probant de considérer que l'archaïsme que I'on rencontre dans ces sépultures de la Basse Époque est la forme que prend la citation artistique dans les tombes de l'Égypte pharaonique quand le décor cite une autre représentation éloignée dans le temps et qui ne lui est pas liée par une tradition continue [76]. On intègrerait alors la créativité ou I'innovation présente dans le phénomène sans devoir recourir à des expressions du type « archaïsme innovant » ou « copie productive ». Inclure ces représentations dans le concept de citation artistique permettrait en outre de ne pas écarter les liens présents entre tombes contemporaines.

Parallèlement, utiliser le concept d'intericonicité [77] pour étudier les liens entre les représentations concernées rendrait compte de l'aspect dynamique de leurs relations et sortirait d'une vision linéaire entre le « modèle » et sa « copie ». En effet, comme l'a explicité Dimitri Laboury, l'intericonicité est « the shaping of an image's meaning or form by another image » [78], mais c'est surtout le cadre conceptuel qui permet de décrire les différentes possibilités d'interactions entre les images de manière dynamique en « taking into account the questions of forms, styles and supports, as well as the levels of consciousness, the intention(s) and the agency of the different actors in the production of images $\gg$ [79].

Afin d'aller vers une meilleure compréhension de ces citations et pour donner au phénomène sa vraie dimension, nous devons nous interroger sur ses possibles caractéristiques, sur les facteurs qui l'influencent et sur les contextes et les acteurs impliqués [80]. Au sein d'une étude typologique des citations artistiques des décors des tombes thébaines privées du Nouvel Empire à la Basse Époque (environ 1550 - 332 av. J.-C.), il faudra en distinguer les caractéristiques formelles, telles que la technique utilisée (peinture, relief), l'échelle de la représentation transmise (paroi, registre, etc. ) [81] et son thème, ainsi que la localisation des représentations citées et citantes.

De même, il faudra envisager le procédé utilisé, tel que I'inversion [82] ou l'ajout d'un personnage, ou encore le redécoupage de la composition. On pourra également discerner les citations qui lient des monuments dits privés de celles réalisées entre un monument royal et un monument privé, les citations qui lient deux monuments voisins de celles qui concernent des monuments éloignés, les citations de monuments contemporains de celles de monuments plus anciens, et au sein de ces dernières, les citations qui s'insèrent dans une tradition continue de celles séparées par un hiatus temporel.

C'est bien souvent une relation professionnelle et/ou familiale entre les défunts ou la proximité de leurs sépultures qui peut expliquer une citation [83], mais les raisons qui sont au cœur des citations artistiques posent bien entendu de nombreuses questions en rapport avec une certaine identité affirmée par le défunt : cherche-t-il à affirmer sa connaissance d'une certaine tradition et ainsi un certain statut social ?
[73] Voir notamment Forero-Mendoza 2004.

[74] DEN DONCKER 2017, p. 346-349.

[75] Pischikova 1998, p. 76-78.

[76] À distinguer des cas où seule la forme, le style sont archaïsants et où le contenu ne renvoie pas à une représentation spécifique.

[77] Voir notamment ARRIVÉ 2015.

[78] LABOURY 2017, p. 248.

[79] Ibid., p. 249.
[80] Dimitri Laboury a énoncé des pistes dans cette direction et des critères d'analyse dans LABOuRY 2013, p. 23.

[81] Rania Merzeban a par exemple proposé trois catégories intéressantes selon le degré de transmission de la scène : « reproduction de parois complètes, reproduction de registres et reproduction de motifs particuliers ». Merzeban 2014, p. 341.

[82] À propos de l'inversion, voir LABOURY 2017, p. 235.

[83] Den Doncker 2017, p. 340. 
Cherche-t-il à démontrer un goût pour une certaine esthétique ? Cherche-t-il à marquer les esprits par un programme décoratif étonnant et ainsi assurer sa pérennité dans la mémoire collective?

Ces différents critères et questions constituent des pistes non exclusives pour une meilleure compréhension de cette forme de transmission et de réinterprétation de la tradition. Dans cette même optique, nous proposons d'avoir recours à la théorie des réseaux ou des graphes, utilisée notamment en sciences humaines et sociales pour étudier la diffusion des innovations ou les réseaux textuels. Plus récemment, cette méthode a aussi été appliquée aux disciplines historiques [84], par exemple pour étudier les mouvements de populations anciennes ou des échanges économiques. Une première approche des citations artistiques dans les tombes thébaines montre qu'elles constituent un réseau très dynamique et complexe que I'application d'une telle méthode permettra de mieux comprendre et de mieux caractériser. Dans un graphe, on peut ainsi considérer les citations comme les arêtes qui lient les nœuds (ou sommets) que constituent les représentations citées et citantes (fig. 1). À partir de là, on peut les quantifier et par exemple identifier des sommets centraux, des sommets isolés, ou des points de passage obligés, de façon à étudier de manière plus précise les voies et les modes de la transmission et de la réactualisation des signifiés et des signifiants iconiques.

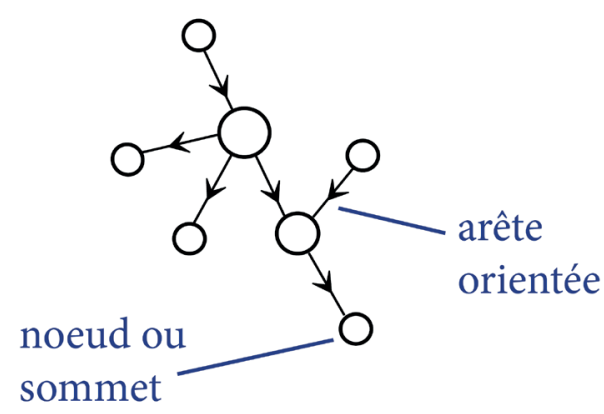

Fig. 1 : Représentation schématique d'un réseau sous forme de graphe. DAO : H. Smets.
Afin de savoir si elles peuvent être transposées avec succès aux citations dans l'art égyptien et nous apporter une plus grande finesse dans la compréhension de celles-ci, nous devons par ailleurs envisager les terminologies et les typologies existantes à propos de ces représentations et citations, à la fois en histoire de l'art égyptien, de l'art gréco-romain et de l'art contemporain.

Ainsi, I'hybridisme, défini par Shelley Wachsmann comme « the phenomenon in Egyptian art by which subjects, be they human figures, objects or even entire scenes, were composed by uniting elements originally belonging to two or more separate entities » [85], pourrait être étendu à une série de cas, en particulier à la Basse Époque, où une sépulture en cite plusieurs autres. Existerait-il des tombes dont le décor est entièrement composé de citations ? C'est I'une des questions que I'on peut poser ici.

Dès lors que la plupart des cas de citation connus à ce stade peuvent être déterminés par un lien entre les propriétaires des tombes concernées, la proposition faite par Alexis Den Doncker d'utiliser l'expression « identifying-copies » pour les images « copied from models that carry with them a strong reference to a specific individual(ity) standing for an ideal socio-professional achievement » [86] est également intéressante car elle met l'accent sur ce lien, bien que I'utilisation du terme « copie » ne soit pas idéale. Par ailleurs, Yannick Chapot, dans un article consacré à la pratique de la citation dans les œuvres de l'Equipo Crónica (collectif du pop art espagnol), relève plusieurs cas et termes intéressants. Il y distingue notamment l'expli-citation de l'impli-citation. La première est une citation que I'on peut aisément remarquer, car elle est nettement marquée dans I'œuvre, par exemple par une mise en relief dans le dessin ou un cadrage différent [87]. La seconde en revanche, ne met pas en avant la citation, voire tend à la masquer. Appelée également citation intégrée, elle est alors plus difficile à percevoir du premier coup d'œil [88]. Dans les décors des sépultures privées égyptiennes, il s'agirait plutôt d'impli-citations. Néanmoins, l'expli-citation ne doit pas totalement être exclue : une citation pourrait, par exemple, être explicite en plaçant un élément dans un thème où il ne se trouve pas habituellement. Le spectateur égyptien antique qui possède le cadre de référence le remarquerait immédiatement, alors que le spectateur moderne pourrait ne pas s'en apercevoir.

[84] Voir Beauguitte 2016 ; Cocaud \& Cellier 2012.

[87] СНАРОт 2012, p. 4-5.

[85] WACHSMANN 1987, p. 4.

[86] Den Doncker 2017, p. 339.

[88] Ibid., p. 7. 
Chapot introduit également la notion du centon pictural : « originellement, le centon est une pièce de poésie ou de prose composée entièrement de vers préexistants ou de fragments pris à un ou plusieurs auteurs » [89]. Il propose d'appliquer ce terme aux représentations qui sont entièrement constituées à partir d'images antérieures. Cette catégorie rejoindrait ainsi I'hybridisme de Shelley Wachsmann. Elle est également assez proche de l'éclectisme de l'art romain qui désigne les œuvres d'art s'inspirant d'au moins deux œuvres différentes, ou encore de sujets ou d'époques différentes [90]. En s'ouvrant aux réflexions des disciplines voisines, on constate donc aisément qu'il existe des cadres d'analyse à explorer en égyptologie.

[89] СНAPOT 2012, p. 8.

[90] PeRrY 2005, p. 111.
En conclusion, la terminologie de l'archaïsme pose encore problème en égyptologie alors qu'il s'agit d'un important phénomène de réutilisation et de création à partir de la tradition. Cette forme de créativité est en réalité bien plus étendue qu'il n'y paraît au sein d'une société que I'on a tendance à percevoir comme figée dans une tradition millénaire transmise uniquement de manière reproductive. En considérant l'archaïsme dans les tombes privées comme l'une des formes possibles de citation artistique, on peut saisir sa vraie dimension et lui donner une définition plus fidèle à la réalité, qui inclut non seulement la créativité, mais aussi plusieurs autres facteurs, tels que la proximité géographique et l'ancrage dans le présent. Il s'agit là d'un premier pas au sein d'une enquête approfondie sur les caractéristiques et les différentes formes de la citation artistique en Égypte pharaonique, qui permettra de mieux comprendre les mécanismes de la transmission du contenu culturel du passé, les raisons qui la sous-tendent, ainsi que les modes de la circulation des représentations et des personnes qui y sont liées.

\section{BIBLIOGRAPHIE}

Aldred, Cyril, 1961, The Egyptians, London.

Aldred, Cyril, 1980, Egyptian art in the days of the Pharaohs $3100-320$ BC, London.

ArrivÉ, Mathilde, 2015, «L'intelligence des images - l'intericonicité, enjeux et méthodes », E-rea 13.1,

[En ligne] URL : http://journals.openedition.org/erea/4620, lien consulté le 20/11/2020.

DOI : 10.4000 /erea.4620

AssmanN, Jan, 1992, Das kulturelle Gedächtnis : Schrift, Erinnerung und politische Identität in frühen Hochkulturen, München.

DOI : $10.17104 / 9783406703409-287$

BeAuguitte, Laurent, 2016, «L'analyse de réseaux en sciences sociales et en histoire : Vocabulaire, principes et limites », dans Rosemonde Letricot, et al. (dir.), Le réseau. Usages d'une notion polysémique en sciences humaines et sociales, Louvain-la-Neuve, p. 9-24.

Bотнmer, Bernard Wilhelm von, 1985, «The Brussels-Brooklyn Statue of Bakenref », dans Paule Posener-Kriéger (dir.), Mélanges Gamal eddin Mokhtar, Le Caire, p. 99-103.

Bотнmer, Bernard Wilhelm von \& RiefstahL, Elizabeth, 1960, Egyptian sculpture of the late period, 700 B.C. to A.D. 100 , An exhibition held at the Brooklyn Museum, 18 October, 1960 to 9 January, 1961, New York.

Brugsch, Heinrich, 1877, Geschichte Aegypten's unter den Pharaonen nach den Denkmälern, Leipzig.

Bruit Zaidman, Louise \& GHerchanoc, Florence, 2006, «Introduction », Ktèma 31, p. 5-16.

BRUnner, Hellmut, 1970, «Zum Verständnis der archaisierenden Tendenzen in der ägyptischen Spätzeit », Saeculum 21, p. $151-161$.

DOI : $\underline{10.7788 / \text { saeculum.1970.21.jg.151 }}$ 
Brunner, Hellmut, 1975, s.v. Archaismus, LÄ I, col. 386-395.

СнAPOT, Yannick, 2012, «La citation picturale : enjeux et problématiques », Cahiers du CELEC 2, p. 1-10.

Cocaud, Martine \& Cellier, Jacques, 2012, «Introduction à l'Analyse des réseaux », dans Martine Cocaud \& Jacques Cellier (dir.), Le traitement des données en histoire et sciences sociales : méthodes et outils, Rennes, p. 347-378.

Dardenay, Alexandra, 2013, «Rome, les Romains et l'art grec», dans Corinne Bonnet \& Florence Bouchet (dir.), Translatio : traduire et adapter les Anciens, Paris, p. 109-138.

Davis, Whitney, 2003, « Archaism and Modernism in the Reliefs of Hesy-Râ », dans John Tait (dir.), Never Had the Like Occurred: Egypt's View of its Past, London, p. 31-60.

DOI : 10.4324/9781315423494-3.

Den Doncker, Alexis, 2017, « Identifying-copies in the private Theban necropolis. Tradition as reception under the influence of self-fashioning processes », dans Todd Gillen (éd.), (Re)productive Traditions in Ancient Egypt, Proceedings of the conference held at the University of Liège, 6th-8th February 2013, Liège, p. 333-370.

Der Manuelian, Peter, 1994, Living in the past: studies in archaism of the Egyptian Twenty-sixth Dynasty, New York. Der Manuelian, Peter, 1998, « Prolegomena zur Untersuchung saitischer "Kopien" », Studien zur altägyptischen Kultur 26, p. 221-246.

Eggebrecht, Arne, 1973, Schlachtungsbräuche im alten Ägypten und ihre Wiedergabe im Flachbild bis zum Ende des mittleren Reiches, München.

Ergner, Diethelm, 1984, Die monumentalen Grabbauten der Spätzeit in der thebanischen Nekropole, Wien.

ENGerT, Michaela, 2015, « Transfer und Iteration bildlicher Darstellungen in ägyptischen Gräbern », dans Eva Christiane Cancik-Kirschbaum \& Anita Traninger (dir.), Wissen in Bewegung: Institution - Iteration - Transfer, Wiesbaden, p. 371-392.

ERMAN, Adolf, 1914, « Saitische Kopien aus Deir el Bahri », Zeitschrift für Ägyptische Sprache und Altertumskunde 52, p. $90-95$.

DOI : 10.1524/zaes.1915.52.1.90

Erman, Adolf, 1934, Die Religion der Ägypter: ihr Werden und Vergehen in vier Jahrtausenden, Berlin. DOI : $10.1515 / 9783111547176$

FAKHRY, Ahmed, 1947, « A report on the Inspectorate of Upper Egypt », Annales du Service des Antiquités de l'Égypte 46, p. 25-54.

FazzinI, Richard, 1972, « Some Egyptian Reliefs in Brooklyn », Miscellanea Wilbouriana 1, New York, p. 33-70.

Forero-Mendoza, Sabine, 2004, «De la citation dans l'art et dans la peinture en particulier », dans Pierre Beylot (dir.), Emprunts et citations dans le champ artistique, Paris, p. 19-31.

Gauthrer, Henri, 1932, Précis de I'histoire d'Egypte par divers historiens et archéologues. Tome premier, Egypte préhistorique, pharaonique et gréco-romaine, Le Caire.

GrandazzI, Alexandre, 2006, « Archéologie romaine : un archaïsme peut en cacher un autre », Ktèma 31, p. 89-96.

Huet, Valérie \& LissarRague, François, 2006, « Style archaïsant/rites archaïques et archaïsation des rites sur les reliefs néo-attiques », Ktèma 31, p. 179-188.

JosephSon, Jack, 2001, s.v. Archaism, Oxford Encyclopedia of Ancient Egypt 1, p. 109-113.

Jurman, Claus, 2009, «From the Libyan Dynasties to the Kushites in Memphis », dans Gérard Broekman et al. (dir.), The Libyan period in Egypt : historical and cultural studies into the 21st-24th dynasties, Proceedings of a conference at Leiden University, 25-27 october 2007, Leuven, p. 113-138.

Jurman, Claus, 2010, «The Trappings of Kingship: Remarks about Archaism, Rituals and Cultural Polyglossia in Saite Egypt », dans Gyory Hedvig (dir.), Aegyptus et Pannonia IV, Acta Symposii anno 2006, Budapest, p. 73-118.

KAHL, Jochem, 2010, s.v. Archaism, UCLA Encyclopedia of Egyptology [En ligne] URL : https://uee.cdh.ucla.edu/articles/archaism, lien consulté le 15/12/2020.

KeEs, Hermann, 1953, Das Priestertum im ägyptischen Staat : vom neuen Reich bis zur Spätzeit, Leiden.

DOI : $10.1163 / 19606028027 \quad 01-49$

LABourY, Dimitri, 2013, «Citations et usages de l'art du Moyen Empire à l'époque thoutmoside », dans Susanne Bickel (dir.), Vergangenheit und Zukunft: Studien zum historischen Bewusstsein in der Thutmosidenzeit, Basel, p. 11-28.

LABOURY, Dimitri, 2017, «Tradition and Creativity : Intericonicity », dans Todd Gillen (éd.), (Re)productive Traditions in Ancient Egypt, Proceedings of the conference held at the University of Liège, 6th-8th February 2013, Liège, p. 229-258.

LÉvi-Strauss, Claude, 1952, « La Notion d'Archaïsme en Ethnologie », Cahiers Internationaux de Sociologie 12, p. 3-25.

MAspero, Gaston, 1887, L'archéologie égyptienne, Paris.

Merzeban, Rania, 2014, «À propos de quelques analogies iconographiques dans les tombes privées », Bulletin de l'Institut français d'archéologie orientale 114/2, p. 339-361. 
MоRкот, Robert, 2003, «Archaism and innovation in art from the New Kingdom to the Twenty-Sixth Dynasty », dans John Tait (dir.), Never Had the Like Occurred: Egypt's View of its Past, London, p. 79-99.

DOI : 10.4324/9781315423494-5

Моккот, Robert, 2007, « Tradition, Innovation and Researching Lybian Past in Kushite and Saite Egypt », dans Harriet Crawford (dir.), Regime change in the ancient Near East and Egypt: from Sargon of Agade to Saddam Hussein, Oxford, p. 141-164.

DOI : $10.5871 / \mathrm{bacad} / 9780197263907.003 .0009$

NAGY, István, 1973, « Remarques sur le souci d'archaïsme en Egypte à l'époque saïte », Acta Antiqua Academiae Scientiarum Hungaricae 21, p. 53-64.

NAviLLE, Edouard, 1901, The temple of Deir El Bahari. Part IV, The shrine of Hathor and the southern hall of offerings: Plates LXXXVII-CXVIII, London.

Navratilova, Hana, 2017, « Thutmoside Graffiti and Thutmoside Traditions », dans Todd Gillen (éd.), (Re)productive Traditions in Ancient Egypt, Proceedings of the conference held at the University of Liège, 6th-8th February 2013, Liège, p. 537-561.

Neurerter, Sabine, 1994, « Eine neue Interpretation des Archaismus », Studien zur altägyptischen Kultur 21, p. $219-254$. Perdu, Olivier, 2018, « La tendance archaïsante en Égypte aux époques tardives : art de la copie ou de l'imitation ? », dans Hanane Gaber et al. (dir.), Imitations, copies et faux dans les domaines pharaonique et de l'Orient ancien, Paris, p. 200-273.

Perry, Ellen, 2005, The aesthetics of emulation in the visual arts of ancient Rome, Cambridge.

Pischiкova, Elena, 1998, « Reliefs from the Tomb of the Vizier Nespakashuty: Reconstruction, Iconography, and Style », Metropolitan Museum of Art Journal 33, p. 57-101.

Pischikova, Elena (dir.), 2014, Tombs of the South Asasif necropolis: Thebes, Karakhamun (TT 223), and Karabasken (TT 391) in the twenty-fifth dynasty, Le Caire.

DOI : 10.5743/cairo/9789774166181.001.0001

RoccATI, Alessandro, 1995, «Rückgriff auf ältere Traditionen im Dekorationsprogramm von TT 27 », dans Jan Assmann et al. (dir.), Thebanische Beamtennekropolen, Neue Perspektiven archäologischer Forschung Internationales Symposion Heidelberg 9.-13.6.1993, Heidelberg, p. 81-84.

Silverman, David (dir.), 2009, Archaism and innovation: studies in the culture of Middle Kingdom Egypt, New Haven. Spalinger, Anthony, 1978, «The Concept of the Monarchy during the Saite Epoch - an Essay of Synthesis », Orientalia 47/1, p. 12-36.

Stammers, Michael, 2009, The Elite Late Period Egyptian Tombs of Memphis, Oxford.

TIRADRItTI, Francesco (dir.), 2008, Egyptian Renaissance: Archaism and the Sense of History in ancient Egypt, Exposition, Museum of fine arts, Budapest, 8 août - 9 novembre 2008, Budapest.

TIRADRITTI, Francesco, 2013, «The cenotaph of Harwa: archaism and innovation », Egyptian Archaeology 43, p. 17-20. Traunecker, Claude, 2014, « The 'Funeral Palace' of Padiamenope (TT 33): Tomb, Place of Pilgrimage, and Library. Current Research », dans Elena Pischikova, Julia Budka \& Kenneth Griffin (éd.), Thebes in the First Millenium BC, Newcastle upon Tyne, p. 205-234.

Valbelle, Dominique, 2005, « Archaïsme (Égypte)», dans Jean Leclant (dir.), Dictionnaire de l'Antiquité, Paris, p. 184-185.

Wachsmann, Shelley, 1987, Aegeans in the theban tombs, Leuven.

WrLson, Penelope, 2010, « Consolidation, Innovation and Renaissance », dans Willeke Wendrich (dir.), Egyptian archaeology, Malden, p. 241-258.

WINAND, Jean, 2017, «(Re)productive traditions in Ancient Egypt. Some considerations with a particular focus on literature and language(s)», dans Todd Gillen (éd.), (Re)productive Traditions in Ancient Egypt, Proceedings of the conference held at the University of Liège, 6th-8th February 2013, Liège, p. 19-40.

Wolf, Walther, 1930, «Zur Auseinandersetzung zwischen der ägyptischen und griechischen Kunst », Archiv für Orientforschung 6, p. 263-273.

Yoyotre, Jean, 1987, « Les arts sous les derniers Libyens », dans Tanis, l'or des pharaons, Marseille, p. 74-75.

ZAGDoun, Mary-Anne, 1989, La sculpture archaïsante dans l'art hellénistique et dans l'art romain du Haut-Empire, Athènes (Bibliothèque des Écoles françaises d'Athènes et de Rome). 